\title{
Die Notaufnahme der Zukunft Aushängeschild der klinischen Versorgung
}

Der Bedarf an akuter medizinischer Versorgung wird immer größer ${ }^{1}$. Notaufnahmen bilden die Schnittstelle zwischen ambulanter und stationärer Versorgung, in der fast alle medizinischen Fachdisziplinen zusammenarbeiten. Doch längst nicht überall tragen Notaufnahmen als eigenständige Organisationseinheiten professionell dem heutigen Ansturm und den Anforderungen seitens der Patienten Rechnung.

\section{Der erste Eindruck prägt: Sorgen und Nöte der Patienten ernst nehmen}

Die Notaufnahme ist das Aushängeschild der Klinik: Häufig findet hier von Seiten der Patienten und deren Angehörigen der Erstkontakt mit der Klinik statt. Die Erfahrungen in der Notaufnahme werden von den Patienten den niedergelassenen Ärzten zugetragen und haben so einen wesentlichen imagebildenden Einfluss im Positiven wie im Negativen. Es gibt wenige Dinge, die bei Patienten einen derart nachhaltig negativen Eindruck zurücklassen, wie der Umstand, mit ihren Sorgen und Nöten in Krisensituationen nicht ausreichend abgeholt zu werden. Unerheblich ist dabei die tatsächliche Diskrepanz der Selbstwahrnehmung der eigenen Situation durch den Patienten sowie der Fremdwahrnehmung durch das entsprechende Fachpersonal. Die fachliche und soziale Kompetenz der Abteilung wird durch sofortige kompetente Ansprache, kontinuierliche und klare Information - insbesondere auch zu den zu erwartenden Wartezeiten - sowie durch empathische Kommunikation gegenüber der Krisensituation, die der Patient empfindet, transportiert. Ein Anspruch, der in

\footnotetext{
'Zwischen 2005 und 2010 wurde eine Steigerung um 16,6\%, d. h. um 2,5 Millionen Behandlungsfälle, auf nunmehr 16 Millionen Menschen verzeichnet, die in Deutschland in einer Notaufnahme behandelt wurden. Das entspricht fast einem Viertel der Gesamtbevölkerung. Gründe sind vielfältig: Veränderung der Altersstruktur in der Gesellschaft, Wegfall von Arztpraxen in ländlichen Regionen, steigendes Gesundheitsbewusstsein und Aufklärung. Vgl. etwa Pressemitteilung des Universitätsklinikums Aachen http://www.ukaachen.de/go/show?ID=170992 38\&ALTNAVID=13319284\&DV=0\&COMP=page \&ALTNAVDV=0 abgerufen am 27.03.2012.
}

der Realität tatsächlich viel zu oft vernachlässigt wird. Dabei bergen gerade diese Punkte großes Potenzial, die Patientenzufriedenheit zu steigern: wenn sich der Patient mit seinen Ängsten und Sorgen und mit kleinen Gesten der Zuwendung gut umsorgt und sicher fühlt, erzeugt das eine Qualität, welche sogar einen Imagegewinn des Krankenhauses mit sich bringen kann.

Wie sieht nun tatsächlich die Realität aus, wenn der Patient mit seinen Ängsten gehend oder liegend in die Notaufnahme kommt? Wird ihm Sicherheit und ein organisierter Eindruck vermittelt, sodass er auf eine erstklassige medizinisch-pflegerische Versorgung schließen kann? Mit der Brille des Patienten gesehen stellt sich dem Betrachter die Organisation der Notaufnahme mitunter recht bekümmernd dar:

- Hektik,

- Patienten werden nach dem Anmelden gefühlt „vergessen“ - am besten noch in einer unfreundlichen Warteecke,

- lange Wartezeiten in Kombination mit fehlenden Informationen an die Wartenden,

- fehlende oder zu wenig an den Patienten angepasste Kommunikation „über ihn, nicht mit ihm“,

- Unklarheiten über Kompetenzen und Zusammenarbeiten der Berufsgruppen äußern sich in Diskussionen im Beisein des Patienten oder der Angehörigen,

- der zuständige Arzt ist aus offensichtlich mangelnder Erfahrung nicht in der Lage, innerhalb einer halben Stunde eine Diagnose oder klare weitere Behandlungsempfehlung auszusprechen, ein zweiter oder gar dritter Arzt wird hinzugezogen. Der Patient ist verunsichert.

Interessant ist, wie viele Patienten hierzu ihre eigenen Geschichten beisteuern können. Dabei hat gerade in den letzten Jahren nicht zuletzt die Nutzung moderner Medien die Patienten in die Lage versetzt, sich eine ganze Reihe an medizinischem Wissen oder Halbwissen anzueignen. Die Zeiten, in denen Patienten mehr oder we- niger kompetenzhörig jeden Ablauf geduldig über sich ergehen ließen, sind vorbei. Heute ist der aufgeklärte, kritische Patient die Regel, der mit klaren Erwartungen in die Klinik kommt. Fallen Erwartung und erlebte Realität auseinander, ist ein Defizit in der Versorgungsqualität offensichtlich und das Vertrauen in die medizinische Kompetenz von Anfang an negativ belastet.

\section{Zentrale Stressfaktoren \\ systematisch lösen}

Umgang mit Belastungsspitzen

Aus unserer Sicht gibt es 3 zentrale Ursachen, warum Patienten in ihren Sorgen und Nöten nicht ernster genommen werden. In deren Zentrum stehen Flexibilität, Quantität und Qualität der Personalbesetzung: Fehlendes Belastungsspitzenmanagement, fehlende unmittelbare Refinanzierung des Personaleinsatzes in der Notaufnahme und Mangel an qualifiziertem Fachpersonal. Den Schlüssel für ein besseres Management von Notaufnahmen sehen wir in der Lösung dieser Engpass- und Stressfaktoren.

Die Gefahr von Patientenschäden ist unter der besonderen Stoßbelastung in der Notaufnahme hoch. Beispiele hierfür sind unvollständige Information zum Patienten, Fehler in der Kommunikation, kognitive Fehler wie z.B. in der Diagnosestellung. Bei punktueller Spitzenbelastung den kritischen Patienten zielsicher aus der Reihe der ambulanten Versorgungsfälle zu filtern und entsprechend einzuordnen, ist wichtig. Gerade unter hoher Stressbelastung wächst jedoch das Risiko diesbezüglicher Vorkommnisse.

Es ist immer wieder zu hören, dass das Patientenaufkommen in einer Notaufnahme nicht planbar ist. Deshalb kann es zur Überschreitung von Behandlungskapazitäten kommen, wodurch die Sicherheit von Patienten gefährdet werden kann. Tatsächlich stellen wir fest, dass die Auswertungen der Uhrzeiten des Patienteneintreffens im Tagesverlauf in Notaufnahmen ähnelnde Muster aufweisen, wie die „ambulante Walflosse in der chirurgischen Notaufnahme“ (Abb. 1).

Wiederkehrende Muster ermöglichen es, mit flexibilisiertem Personaleinsatz ein systematisches Belastungsspitzenmanagement zu betreiben. Spitzenauslastungen gegen 11 Uhr sind also nicht jeden Tag aufs Neue wieder eine Überraschung. Tatsächlich häuft sich die Behandlung der Patienten in engen Zeitkorridoren, z.B. 
entfallen 62\% der Fälle des Tages auf einen Zeitraum von 4 Stunden. Eine darauf abgestimmte Personaleinsatzplanung führt zu einer überproportionalen Stressreduktion.

Bewusste Investition statt Abwärtsspirale des Refinanzierungsdiktats

In weniger auf die Notfallversorgung ausgelegten Kliniken wird der Aufnahmebereich gerne nur mit ärztlichen Springerdiensten besetzt, weil für die Vorhaltung eines Arztes in der Notaufnahme keine Vergütung stattfindet. Die Ambulanzpauschalen sind in aller Regel so gering, dass sie nicht einmal annähernd die direkt in dem Bereich anfallenden Kosten abdecken. Dabei kommt der Großteil der chirurgischen Patienten tatsächlich mit leichten Wunden in die Notaufnahme die für die Patienten jedoch durchaus dramatisch sind. Doch auch im internistischen Bereich ist der Anteil ambulanter Notfallbehandlungen groß. Nicht selten ist der Teufelskreis der, dass unerfahrene Ärzte dorthin zum Lernen abgestellt werden und zur eigenen Absicherung einen überdimensionalen Aufwand an Diagnostik anstoßen. Das ist „Learning by Doing“, statt von einem erfahrenen Kollegen an der Seite strukturiert begleitet zu werden. Die Notaufnahme stellt jedoch auch einen Hauptzubringer ökonomisch wichtiger Krankenhausfälle dar. Ein organisierter Notfallbereich hat somit einen unmittelbaren, wesentlichen Einfluss auf die Wirtschaftlichkeit der Gesamteinrichtung. Je höher das Zutrauen in die Einrichtung, desto höher wird auch der Zufluss an „angemessen“ vergüteten stationären Fällen sein. Entscheidend ist es also, das richtige Fachpersonal in der Notaufnahme zu haben, das im akuten Bedarfsfall den vital gefährdeten Patienten sofort und medizinisch adäquat versorgt. Ideal ist folglich eine professionell von einem - disziplinarisch fachabteilungsungebundenen - Facharzt zusammen mit einer korrespondierenden pflegerischen Leitung geführte, eigenständige Notfallaufnahmeeinheit. Eine Investition in die Notaufnahme ist entsprechend auch eine Investition in die Zukunft der Klinik. Tatsächlich ist diese Qualität im heutigen Finanzierungssystem jedoch allenfalls an ausgemachten Notfallversorgungsstandorten unmittelbar oder mittelbar refinanziert. Die Frage ist, ob sich eine Klinik ein weniger an Qualität auf Dauer wirklich leisten kann?

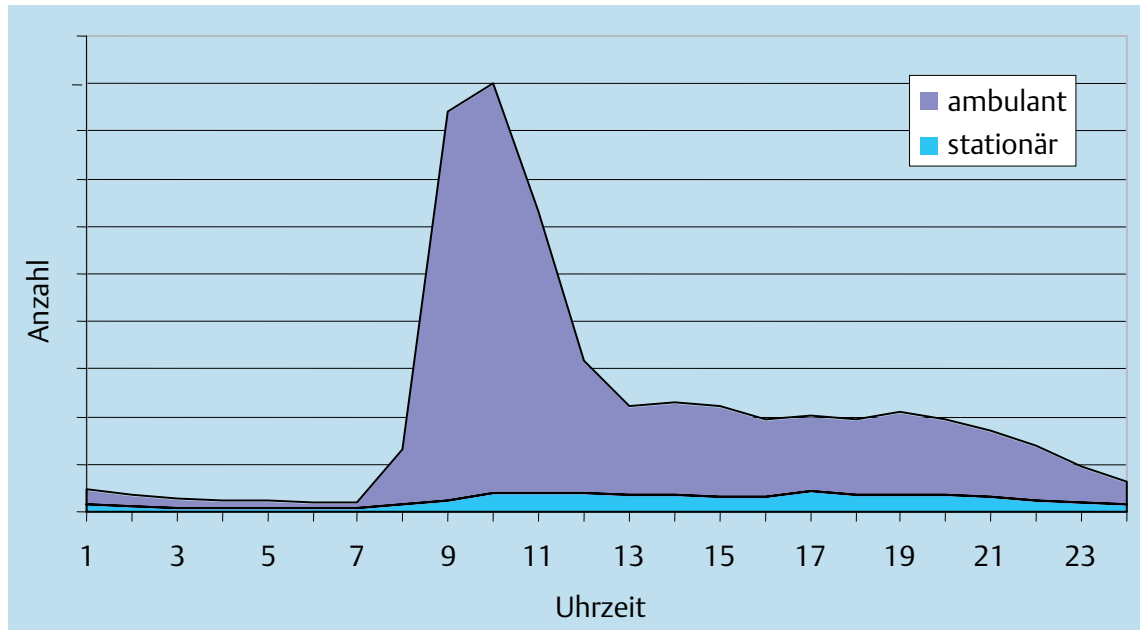

Abb. 1 Patienteneintreffen in einer chirurgischen Notaufnahme nach Uhrzeit (Quelle: eigene Berechnungen).

Kontinuierliche Entwicklung qualifizierten Fachpersonals

Die Notaufnahme hält, vielleicht zusammen mit der Intensivpflege, das wohl am vielfältigsten gefächerte Anforderungsprofil an das Personal bereit. Multidisziplinäres fachliches Können (u.a. Innere Medizin, Chirurgie, Anästhesie), gepaart mit ausgeprägten sozialen Fähigkeiten wie Empathie und Kommunikation, ergänzen das Bild der geforderten „eierlegenden Wollmilchsau“ in der Notaufnahme. Hier obliegt dem Managementteam der Notaufnahme im besonderen Maße die Aufgabe, die Mitarbeiter entsprechend zu entwickeln und den Mitarbeitern ihren Raum im multiprofessionellen Team zuzuweisen. Damit die Organisationseinheit nachhaltig von der hohen Qualität des vorhandenen Personals profitiert, sind eine strukturierte Personaleinarbeitung sowie kontinuierliche Personal- und Teamentwicklung elementare Säulen in der Mitarbeiterführung und in Zeiten des gravierenden Fachkräftemangels auch Instrumente der Mitarbeitergewinnung und -bindung.

Ein funktionierendes Team, das fachlich und sozial hohen Kompetenzanforderungen standhält, unter Stress den Kopf behält und Hand-in-Hand arbeitet, ist daher nicht nur, aber besonders im Notfallversorgungsbereich ein wichtiges Kapital der Klinik. Empathie, Freundlichkeit, Kommunikations-, Team-, Kritik- und Konfliktfähigkeit, Zuverlässigkeit bei gleichzeitiger professioneller Distanz und stressausgleichendem Humor sollten noch viel stärker vom Leitungsteam gefördert werden, als dies heute in der zumeist personell dürftig besetzten Notaufnahme üblich ist.

\section{Verantwortlichkeiten klären und Verbindlichkeit herstellen}

Um die Prozesse in der Notaufnahme zu strukturieren, Verantwortlichkeiten zu definieren und Verbindlichkeiten festzuschreiben, ist der erste Schritt, ein Organisationskonzept auszuarbeiten, welches die wichtigsten Prozesse und Regelungen beschreibt.

Die Arbeitszeit der erfahrensten Mitarbeiter ist kostbar. Entsprechend sollten gerade für diese Mitarbeiter Freiräume des ununterbrochenen Arbeitens geschaffen werden und unnötige Wegezeiten vermieden werden. Das Arbeiten der Berufsgruppen soll gut verzahnt funktionieren und systematische Abstimmungen bei wechselndem Personal nicht täglich neu getroffen werden. Die berufsgruppenübergreifenden Abstimmungen $\mathrm{zu}$ aufbau- und ablauforganisatorischen Fragestellungen lassen sich in einem Handbuch für die Notaufnahme dokumentieren. Die organisatorischen Aufgaben und Strukturen einer Notaufnahme und der Beitrag des Einzelnen zum Ganzen werden transparent. Damit schafft es eine Basis, Committment über arbeitsteilige Abläufe im Funktionsbereich mit klaren Verantwortlichkeiten zu erreichen.

Ein Handbuch organisiert die wichtigsten Prozesse, regelt Verantwortlichkeiten, fördert Verbindlichkeiten und ist zudem ein hilfreiches Dokument zum Einarbeiten neuer Mitarbeiter. Hierarchische Strukturen und deren uneingeschränkte Akzeptanz durch alle Beteiligten im Prozess sind gerade im „Krisenmanagement“ wichtig, um die Stressbelastung der Mitarbeiter zu reduzieren bzw. die Sicherheit zu erhöhen. Gleichzeitig ist eine Hierar- 
chie „nach Befehl und Gehorsam“ gerade in zeitkritischen Prozessen ineffektiv, sollen doch die situativ besten Entscheidungen und Lösungen zeitnah herbeigeführt werden. Die Rollenübernahme im Team ist dementsprechend zu klären und bedingt eine Unterordnung unter ein gemeinsames Ziel, wenn die Integration von Fachexperten in einem durch Sicherheit und Vertrauen geprägten Teamklima gelingen soll. Dafür muss das Handbuch auch nach seiner Einführung ein immer wieder diskutiertes Instrument bleiben (s. Kasten).

Die Stichworte auf der Liste der ablauforganisatorischen Konzepte sind im Detail beliebig vielfältig. So hat sich gerade in den nördlichen Regionen das System der Triagierung schon weitgehend durchgesetzt. Gerade die pflegerische Triage leistet eine zuverlässige Erstsichtung der Patienten und filtert so Versorgungsrisiken heraus. Gleichzeitig wird das ärztliche Personal entlastet. So kann eine zügige und systematische Patientenbehandlung in der Notaufnahme besser gewährleisten werden. Das Triagierungssystem bietet den organisatorischen Rahmen, Notfallalgorithmen mit Schnittstellenpartnern der unterschiedlichen Kliniken abzubilden. Die Notfallbehandlungsabläufe können in Diagnostikprofile und Behandlungsalgorithmen weiter segmentiert werden und so Prozesse zur Diagnosefindung und Therapie abbilden. Die begleitende Einführung eines strukturierten Risikomanagements zur Erhöhung von Patienten- und Diagnosesicherheit kann die Fehlerkultur und Behandlungsqualität der Organisationseinheit verbessern. Die Notaufnahmen sind zum gegenwärtigen Zeitpunkt schon stark frequentiert und werden in der Zukunft noch weiter wachsen. Eine Auseinandersetzung mit Managementansätzen und Finanzierungsansätzen im Notfallbereich ist längst überfällig. So liegt Potenzial in wirtschaftlicher wie auch in qualitativer Sicht an der intersektoralen Schnittstelle zwischen ambulanter und stationärer Medizin brach. Aber auch das Management der medizinisch-pflegerischen Kernprozesse innerhalb der Klinik ist in zukunftsfähigen Notfallversorgungsstrukturen maßgeblich professionalisiert. Die Ansätze konzentrieren sich strukturell auf 3 Dimensionen:

- klare, interprofessionell definierte Strukturen innerhalb des Notaufnahmeteams,

\section{Handbuch der Zentralen Notaufnahme Inhaltsverzeichnis}

1 Ziele und Regelungsbereiche des Organisationshandbuches ..........................2

2 Personelle Struktur und Besetzung der Notaufnahme................................... 3

2.1 Organigramm der Notaufnahme ......................................................... 3

2.2 Besetzung \& Rahmendienstplanung ................................................... 4

2.3 Interdisziplinäre Alarmierungsketten...................................................... 4

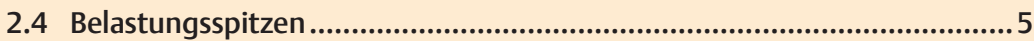

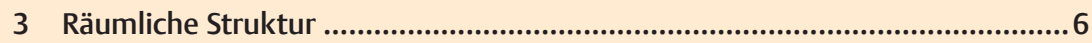

3.1 Patientenleitsystem und Zugangswege ............................................6

3.2 Raumnutzungsplan........................................................................ 8

4 Übersicht zur berufsgruppenübergreifenden Prozesslandschaft..................... 10

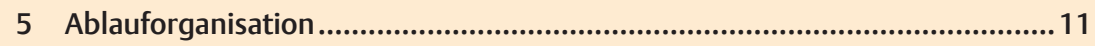

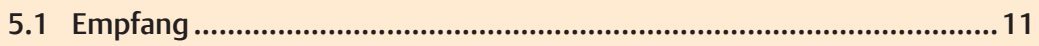

5.2 Pflegerische Triagierung ................................................................ 11

5.3 Wartezimmer-Management .......................................................... 13

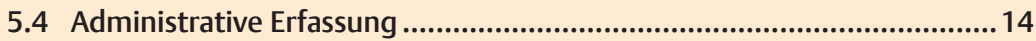

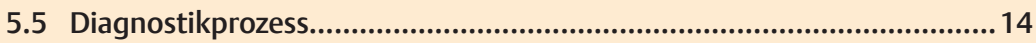

5.6 Dokumentation \& Befundbericht........................................................ 16

5.7 Verlegungen auf die Aufnahmestation/ Stationen ................................. 17

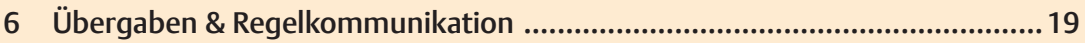

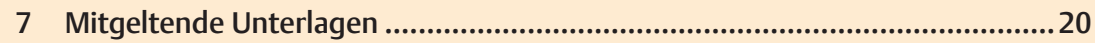

(Quelle: eigene Darstellung)

- klare, interdisziplinäre Schnittstellenvereinbarungen mit den Fachbereichen,

- klare Dienstleistungsvereinbarungen mit angrenzenden medizinischen Serviceeinheiten (Diagnostikzentrum, Belegungsmanagement).

Ein professionelles Management der Notaufnahmebereiche ist unabdingbar, um Verbindlichkeit und Professionalität in diesem Bereich zu erreichen. Aufgrund der Vielzahl an Schnittstellen zur berufsgruppen- und fachübergreifenden $\mathrm{Zu}$ sammenarbeit mit entsprechendem Einfluss auf die Wirtschaftlichkeit der Patientenversorgung ist ein reines Notaufnahmemanagement jedoch nicht hinreichend. Es müssen in diesem Kontext viele Faktoren beachtet werden: Es geht um wirkungsvolle Kommunikationsstrukturen, die Minimierung von Informationsverlusten, Zeitverlusten und Personalbindung, das Erzielen von Verbindlichkeiten innerhalb „matrixorganisatorisch" strukturierter Arbeitsbereiche mit begrenzten disziplinarischen Mitteln bis hin zu gemeinsamen Fortbildungen. Die Notaufnahme erhält Bedeutung für die Patientenklassifikation nicht nur in Bezug auf die Behandlungsdringlichkeit, sondern auch im Hinblick auf die Entscheidung eines ambulanten, normalstationären oder intensivmedizinischen Be- handlungspfades bezüglich notwendiger interdisziplinärer Eingangsassessments (z.B. Behandlungspfad geriatrisches Komplexbehandlung) und auch im Hinblick auf eine weitgehende Fachabteilungszuordnung ohne Fachbereichsegoismus. Sorgen und Nöte der Patienten an dieser Stelle im Ganzen ernst zu nehmen, heißt nicht zuletzt, hier für eine interprofessionell und interdisziplinär unabhängige und straffe Führung zu sorgen.

\section{Korrespondenz}
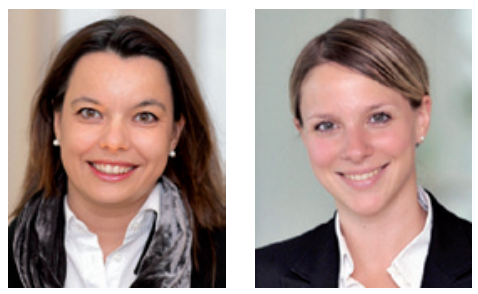

Dr. Elke Eberts Ruhl Consulting AG Alexa Schmall Harrlachweg 68165 Mannheim

E-Mail: info@ruhl-consulting.de

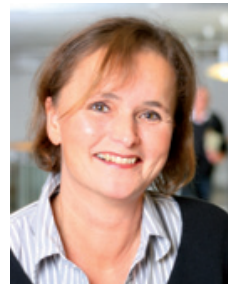

Nicole Giese

Pflegeleitung Aufnahmezentrum, Schulstation und Zentraler Transportdienst FEK Friedrich-Ebert-Krankenhaus $\mathrm{GmbH}$ Neumünster Friesenstraße 11

24534 Neumünster 